\title{
Life cycle cost analysis considering multiple dependent degradation processes and environmental influence
}

\author{
Bin Liu ${ }^{1}$, Xiujie Zhao ${ }^{2}$, Guoquan $\mathrm{Liu}^{3}$, and Yiqi Liu*4 \\ ${ }^{1}$ Department of Management Science, University of Strathclyde, Glasgow, UK \\ ${ }^{2}$ Department of Systems Engineering and Engineering Management, City University of Hong Kong, \\ Kowloon, Hong Kong \\ ${ }^{3}$ International Business School Suzhou, Xian Jiaotong-Liverpool University, Suzhou, 215123, China \\ ${ }^{4}$ School of Automation Science and Engineering, South China University of Technology, Guangzhou,
} China

\begin{abstract}
This paper presents a life cycle cost model for systems subject to multiple dependent degradation processes and environmental influence. Different from most existing studies that assume an infinite time horizon for cost analysis, we evaluate the life cycle cost within a finite horizon. The system is subject to multiple degradation processes, among which the dependence is described via copula. In addition to the stochastic dependence of the degradation processes, the health condition of the system is influenced by the operating environment. The environmental influence on the system condition lies in two aspects: It accelerates or decelerates the degradation processes and meanwhile leads to a random failure threshold. System reliability considering multiple dependent degradation processes and environmental influence is assessed

*Corresponding author. E-mail: aulyq@scut.edu.cn
\end{abstract}


as the first step, followed by the life cycle cost analysis. A numerical example is presented to illustrate the proposed model.

KEY WORDS: Life cycle cost analysis; multiple degradation processes; stochastic dependence; copula; reliability analysis

\section{Introduction}

Life cycle cost analysis involves a number of elements, such as cost of initial construction, maintenance, operation, and many other actions, over the specific service life of a system. As reported in reliability-based optimization, the three most significant elements of such cost analysis are the cost of construction, benefits from system service and economic losses due to failures or performance degradation (Rosenblueth and Mendoza, 1971). In terms of economic losses due to failures, cost estimation uncertainty mainly arises from the intrinsic degradation process, for instance, estimation uncertainty of the degradation path and the associated parameters, as well as the impact of the operating environment.

Continuous improvements of product reliability have prompted the development of degradation modelling. With the increase of product lifetime, obtaining failure data in a feasible period is difficult, which impedes the application of traditional failure-data-based reliability models. On the other hand, with the advances in inspection and monitoring technologies that can effectively provide information on the system health condition, degradation modelling is widely adopted as an alternative (Lu and Meeker, 1993; Liu, Yeh, Xie and Kuo, 2017). In the area of reliability and maintenance optimisation, stochastic-processes-based degradation models have gained popularity, because of their flexibility in characterising performance deterioration and interpreting failure mechanisms (Liu, Wu, Xie and Kuo, 2017; Liu, Liang, Parlikad, Xie and Kuo, 2017; Zhai and Ye, 2017; Do et al., 2019). In the literature, numerous degradation processes have been proposed, 
among which, the Wiener, gamma and inverse Gaussian processes are most widely used to characterise the system deterioration, owing to their attractive mathematical property and clear physical interpretation (Ye and Xie, 2015).

Despite the efforts on degradation modelling, an implicit assumption of such studies is that the system is subject to a single degradation process. However, as modern systems become increasingly complex for performing multiple functions, there usually exist multiple performance indicators. Each performance indicator may imply an underlying degradation process (Zhao et al., 2018; Gao et al., 2019; Yang et al., 2018). Therefore, bivariate or multivariate stochastic models are preferred to describe the degradation process of such complex systems. Examples of multiple degradation processes can be found in electronic products, heavy machine tools and piping systems (Peng et al. 2016; Pandey et al. 2011). For example, as regards the piping system used for heat transfer in nuclear power plants, the pipe thickness is reduced by corrosive flows and various parts of such pipes become corroded. In addition, the corrosion processes are highly dependent, because the corrosive water usually flows through these parts. For such systems, a single degradation process fails to accurately characterise the underlying corrosion mechanism.

In literature, several studies have been conducted on reliability analysis and maintenance decisionmaking for systems with multiple degradation processes. Liu et al. (2013) developed a conditionbased maintenance policy for a degrading system with multiple failure modes, where average availability and long-run cost rate are formulated as the objective function. Liu et al. (2014) further investigated the criticality measures for a multi-component system with multiple degradation processes. Moreover, several reliability models were established considering multiple dependent competing failure processes in previous works (Song et al., 2014; Jiang et al., 2012; Ye et al., 2011; Wang et al., 2011; Hong et al., 2014; Riascos-Ochoa et al., 2016). Notably, copula methods have played an increasingly important role in describing interdependence among the degradation processes. Pan et al. (2013) addressed the issue of parameter estimation for a system subject to a bivariate Wiener process, using copula method to characterise the dependence of the degradation 
processes. Peng et al. (2016) proposed a bivariate degradation model based on an inverse Gaussian process and copulas and implemented a two-stage Bayesian method for parameter estimation under incomplete degradation observations. Wang and Pham (2012) developed a reliability model considering multiple dependent competing failures and employed time-varying copulas to fit the dependence relationship among the degradation processes.

Although several attempts have been made for cost analysis considering multiple degradation processes, these mainly focus on the asymptotic cost rate (cost per unit time). The reason for its popularity is that the asymptotic cost rate can be simply computed with the renewal cycle theorem, which states that the long-run cost rate equals the expected cost in a renewal cycle divided by the expected length of a renewal cycle. The asymptotic criterion assumes that the system can operate in an infinite time horizon. However, in practice, the application of this asymptotic property is questionable for most engineering systems, which exhibit a finite operating horizon (Qiu et al. 2018; Sánchez-Silva et al. 2016). In addition, with the rapid development of technology, many products (e.g.,electronic products) are updated frequently and assuming an infinite usage horizon is unrealistic. Therefore, we propose to investigate the damage cost under the framework of a finite life cycle. Previous life-cycle studies mostly focus on shock models or a single degradation process. For example, Pandey et al. (2011) analysed the maintenance cost of a degrading system over a finite horizon. The adopted the gamma process to describe the degradation process and obtained both expectation and variance of the cost for future decision-making. Pandey and van der Weide (2017) developed a stochastic renewal process model to estimate the life-cycle damage cost for a structural system, proposing a a renewal decomposition method to solve a range of renewal cycle issues. Frangopol (2011) presented an overview of life-cycle management and optimisation under aleatory and epistemic uncertainties. Caballé and Castro (2017) proposed a reliability model for finite life-cycle systems subject to degradation and shock. Yet in that work, the authors considered only one degradation process. Sanchez-Silva et al. (2011) investigated the life-cycle performance of structures considering multiple sources of degradation. However, in that work, the authors took 
account of the combination of progressive degradation and sudden events, while the influence of multiple continuous degradation processes warrants further investigation. In addition, many factors of the operating environment, such as temperature, humidity and pressure, may impose an impact on the degradation process (Gebraeel and Pan, 2008; Liu et al., 2014). Operating in an extreme environment may aggravate the deterioration and reduce the lifetime of the system. Therefore, it is important to take into account the environmental influence when performing life-cycle cost analysis. However, study on life-cycle cost analysis in presence of multiple degradation processes and environmental influence remains wide open.

In this study, we aim to investigate the life-cycle cost of a system under multiple degradation processes and environmental influence. The life-cycle cost in this study refers to the total cost incurred owing to service loss, system damage and restoring operations of the failed system. Although several previous studies have considered the multiple degradation processes for life-cycle cost analysis, they have not addressed the influence of multiple continuous degradation processes and the operating environment. We aim to fill the research gap by providing a comprehensive life-cycle cost analysis framework for systems with multiple dependent degradation processes and environmental influence. We use gamma process to model the degradation process and characterise the dependence via copula. We investigate the evolution of system reliability as a first step, which establishes the basis for life cycle analysis. In addition to obtaining the expected life cycle cost, we also investigate the variance to provide additional insights for financial planning and capital budgeting.

The remainder of this paper is organised as follows: Section 2 presents the reliability model incorporating the dependent degradation processes and environmental influence. Life cycle cost analysis is performed in Section 3. Section 4 provides a numerical example to illustrate the proposed model. Finally, concluding remarks and future research are provided in Section 5. 


\section{Nomenclature}

\begin{tabular}{|c|c|}
\hline$I$ & Number of degradation processes \\
\hline$X_{i}(t)$ & $i$ th degradation process, $i \in\{1,2, \ldots, I\}$ \\
\hline$G a\left(t ; \alpha_{i}, \beta_{i}\right)$ & Gamma process with shape parameter $\alpha_{i}$ and scale parameter $\beta_{i}$ \\
\hline$T_{i}$ & Time to failure of the $i$ th degradation process \\
\hline$\zeta_{i}$ & Failure threshold of the $i$ th degradation process \\
\hline$C(\bullet)$ & Copula function \\
\hline$c(\bullet)$ & Copula density function \\
\hline$K(t)$ & Total discounted cost in time interval $(0, t)$ \\
\hline$S_{i}$ & $i$ th renewal cycle \\
\hline$\rho$ & Discount rate \\
\hline$C_{i}$ & Cost at the $i$ th renewal cycle, with mean $\mu_{c}$ and standard deviation $\sigma_{c}$ \\
\hline$N(t)$ & Number of renewal cycles in time $(0, t)$ \\
\hline$T_{f}$ & Time to failure of the system \\
\hline$E\left[K^{2}(t)\right]$ & Second moment of the discounted cost \\
\hline $\operatorname{Var}(K(t))$ & Variance of the discounted cost \\
\hline$\Lambda(t)$ & Renewal function, $\Lambda(t)=E[N(t)]$ \\
\hline$F_{X_{1}, X_{2}, \ldots, X_{I}}\left(x_{1}, x_{2}, \ldots, x_{I} ; t\right)$ & Joint distribution of the degradation levels \\
\hline$R(t)$ & System reliability function \\
\hline
\end{tabular}

\section{System reliability modelling}

In this section we investigate system reliability considering multiple dependent degradation processes and environmental influence. As a first step we analyse the individual degradation processes and obtain the marginal lifetime distribution with respect to these processes. Copulas are employed 
subsequently to describe the dependence among the degradation processes. Then we step forward to investigate the environmental influence by introducing the covariates and random failure thresholds. Compared with other modelling approaches, the incorporation of covariates and random failure thresholds makes it more flexible to incorporate external influences.

\subsection{Individual degradation process without environmental influence}

Consider a system subject to $I$ degradation processes. Let $\left\{X_{i}(t), t>0\right\}$ denote the $i$ th degradation process, $i \in\{1,2, \ldots, I\}$. It is assumed that without environmental influence, each degradation follows a stationary gamma process, with shape parameter $\alpha_{i}$ and scale parameter $\beta_{i}$, $X_{i}(t) \sim G a\left(t ; \alpha_{i}, \beta_{i}\right)$. We consider the homogeneous Gamma process as it is one of the most widely used stochastic processes that are applied in degradation modelling. In addition, employment of the homogeneous Gamma process makes it accessible to analytical results for system reliability evaluation and life cycle cost analysis. It is well known that gamma process exhibits the property of independent increments. Between two time epochs $t$ and $l(t>l)$, the degradation increment, $\Delta X_{i}(t-l)=X_{i}(t)-X_{i}(l)$, follows a gamma distribution, with the probability density function (pdf) and cumulative distribution function (cdf) as

$$
f_{\Delta X_{i}}(x ; t-l)=\frac{\beta_{i}^{\alpha_{i}(t-l)}}{\Gamma\left(\alpha_{i}(t-l)\right)} x^{\alpha_{i}(t-l)-1} e^{-\beta_{i} x}
$$

and

$$
F_{\Delta X_{i}}(x ; t-l)=\frac{\gamma\left(\alpha_{i}(t-l), \beta_{i} x\right)}{\Gamma\left(\alpha_{i}(t-l)\right)}
$$

where $\gamma\left(\alpha_{i}(t-l)\right)$ is the lower incomplete gamma function. Without loss of generality, the initial degradation is assumed to be $0, X_{i}(0)=0, \forall i \in\{1,2, \ldots, I\}$. The system fails when any of the degradation levels $X_{i}(t)$ exceeds the associated threshold $\zeta_{i}$. Denote $T_{i}$ as the time to failure in 
terms of the $i$ th degradation process. For an individual degradation process, the cdf of $T_{i}$ is given as

$$
F_{T_{i}}(t)=P\left(X(t) \geq \zeta_{i}\right)=\int_{\zeta_{i}}^{\infty} f_{X_{i}}(x ; t) d x=\frac{\Gamma\left(\alpha_{i} t, \beta_{i} \zeta_{i}\right)}{\Gamma\left(\alpha_{i} t\right)}
$$

where $\Gamma\left(\alpha_{i} t, \beta_{i} \zeta_{i}\right)$ is the upper incomplete gamma function. The associated pdf is expressed as

$$
f_{T_{i}}(t)=\frac{\alpha_{i}}{\Gamma\left(\alpha_{i} t\right)} \int_{\beta_{i} \zeta_{i}}^{\infty}\left(\log (z)-\psi\left(\alpha_{i} t\right)\right) z^{\alpha_{i} t-1} e^{-z} d z
$$

where $\psi(y)=\Gamma^{\prime}(y) / \Gamma(y)$ is the digamma function.

\subsection{Modelling system reliability with copula}

Traditionally, a commonly used method for dependence modelling is via a multivariate distribution, which, however, suffers the limitation of intractability. As an alternative, in this section, we use copula functions to describe the joint distribution of the degradation processes. Dependence modelling via copula exhibits several advantages over other approaches (Wang and Pham, 2012; Nelsen, 2007). A copula function allows us to separately model the marginal distributions and the structure of dependence, in which the marginal distributions can be of any form without restriction. In addition, copula functions remain invariant under increasing and continuous transformation, which provides more flexibility to describe the joint distribution.

Denote $\left(X_{1}(t), X_{2}(t), \ldots, X_{I}(t)\right)$ as the $I$-dimensional random vector of the degradation levels at time $t$ and their joint distribution as $F_{X_{1}, X_{2}, \ldots, X_{I}}\left(x_{1}, x_{2}, \ldots, x_{I} ; t\right)$, i.e.,

$$
F_{X_{1}, X_{2}, \ldots, X_{I}}\left(x_{1}, x_{2}, \ldots, x_{I} ; t\right)=P\left(X_{1}(t) \leq x_{1} \cap X_{2}(t) \leq x_{2} \cap \ldots \cap X_{I}(t) \leq x_{I}\right)
$$


The joint distribution can be expressed in the form of a copula function,

$$
F_{X_{1}, X_{2}, \ldots, X_{I}}\left(x_{1}, x_{2}, \ldots, x_{I} ; t\right)=C\left(F_{X_{1}}\left(x_{1} ; t\right), F_{X_{2}}\left(x_{2} ; t\right), \ldots, F_{X_{I}}\left(x_{I} ; t\right)\right)
$$

where $C:[0,1]^{I} \rightarrow[0,1]$ is the copula function. Based on Sklar's theorem, since $F_{X_{i}}\left(x_{i} ; t\right)$ is continuous, the copula function $C$ is unique (Nelsen, 2007). The associated pdf of the joint distribution is given as

$$
\begin{aligned}
& f_{X_{1}, X_{2}, \ldots, X_{I}}\left(x_{1}, x_{2}, \ldots, x_{I} ; t\right)= \\
& c\left(F_{X_{1}}\left(x_{1} ; t\right), F_{X_{2}}\left(x_{2} ; t\right), \ldots, F_{X_{I}}\left(x_{I} ; t\right)\right) \prod_{i=1}^{I} f_{X_{i}}\left(x_{i} ; t\right)
\end{aligned}
$$

where $c(\bullet)$ is the copula density function,

$$
c\left(F_{X_{1}}\left(x_{1} ; t\right), F_{X_{2}}\left(x_{2} ; t\right), \ldots, F_{X_{I}}\left(x_{I} ; t\right)\right)=\frac{\partial C\left(F_{X_{1}}\left(x_{1} ; t\right), F_{X_{2}}\left(x_{2} ; t\right), \ldots, F_{X_{I}}\left(x_{I} ; t\right)\right)}{\partial F_{X_{1}} \partial F_{X_{2}} \ldots \partial F_{X_{I}}}
$$

Knowing the marginal distributions will not automatically lead to the optimal fit of a copula. In fact, numerous joint distributions can be derived for given marginal distributions. Therefore, we first need to select a suitable function before applying copulas for modelling the dependence. In the literature, several parametric families have been developed for the modelling purpose, among which the most widely used ones are the Elliptical family (e.g., Gaussian and $t$ copulas) and Archimedean family (e.g., Clayton, Gumbel, Frank and Joe copulas)(Wu, 2014). Figure 1 illustrates the pdf plots of Clayton and Gumbel copulas.

If the Gaussian copula is adopted, the joint distribution can be obtained as

$$
\begin{aligned}
& F_{X_{1}, X_{2}, \ldots, X_{I}}\left(x_{1}, x_{2}, \ldots, x_{I} ; t\right)= \\
& \Phi_{I}\left(\phi^{-1}\left(F_{X_{1}}\left(x_{1} ; t\right)\right), \phi^{-1}\left(F_{X_{2}}\left(x_{2} ; t\right)\right), \ldots, \phi^{-1}\left(F_{X_{I}}\left(x_{I} ; t\right)\right)\right)
\end{aligned}
$$



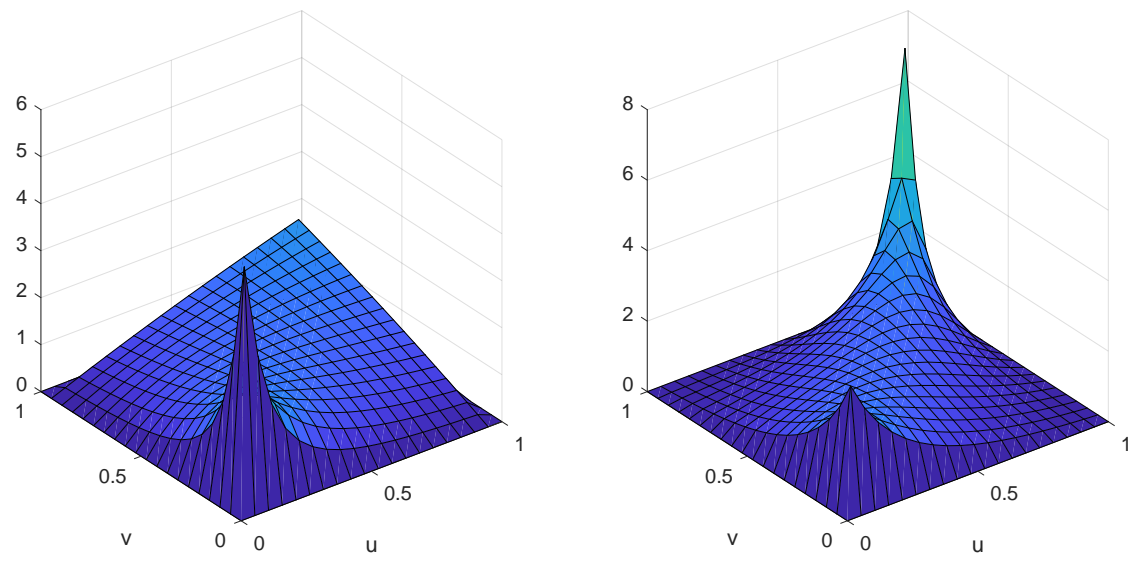

Figure 1: Sketch of Clayton and Gumbel copulas

where $\phi(\bullet)$ is the cdf of the standard normal distribution, and $\Phi_{I}(\bullet)$ is the cdf of a multivariate normal distribution with mean 0 and covariance matrix $\Sigma$. The associated pdf is given as

$$
f_{X_{1}, X_{2}, \ldots, X_{I}}\left(x_{1}, x_{2}, \ldots, x_{I} ; t\right)=\prod_{i=1}^{I} f_{X_{I}}\left(x_{I} ; t\right)|\mathbf{\Sigma}|^{-1 / 2} \exp \left\{-\frac{A(\boldsymbol{\Sigma}-\mathbf{I}) A^{T}}{2}\right\}
$$

where $A$ is an $I$-dimensional vector,

$$
A=\left[\phi^{-1}\left(F_{X_{1}}\left(x_{1} ; t\right)\right), \phi^{-1}\left(F_{X_{2}}\left(x_{2} ; t\right)\right), \ldots, \phi^{-1}\left(F_{X_{I}}\left(x_{I} ; t\right)\right)\right]
$$

Following the Clayton copula, we can have the joint distribution as

$$
F_{X_{1}, X_{2}, \ldots, X_{I}}\left(x_{1}, x_{2}, \ldots, x_{I} ; t\right)=\max \left(\left(\sum_{i=1}^{I} F_{X_{i}}\left(x_{i} ; t\right)^{-\eta}-I\right)^{-1 / \eta}, 0\right)
$$

where $\eta \in[-1,0) \cup(0, \infty)$. The above two copula functions are presented for illustration purpose. One may refer to Nelsen (2007) for more copula functions. Given the initial degradation level 
$X_{i}(0)=0, \forall i \in\{1,2, \ldots, I\}$, the system reliability at time $t$ is given as

$$
\begin{aligned}
R(t) & =P\left(\sup _{s \leq t} X_{1}(s) \leq \zeta_{1}, \sup _{s \leq t} X_{2}(s) \leq \zeta_{2}, \ldots, \sup _{s \leq t} X_{I}(s) \leq \zeta_{I}\right) \\
& =C\left(F_{X_{1}}\left(\zeta_{1} ; t\right), F_{X_{2}}\left(\zeta_{2} ; t\right), \ldots, F_{X_{I}}\left(\zeta_{I} ; t\right)\right)
\end{aligned}
$$

\subsubsection{Estimation of copula parameters}

Let $x_{i j}$ be the degradation measurement for the $i$ th degradation process at time $t_{j}$. Denote $\mathbf{X} \in R^{I \times J}$ as the data matrix, $\mathbf{X}=\left[x_{i, j}\right]$, for $i=1,2, \ldots, I$ and $j=1,2, \ldots, J$. Let $\Delta X_{i, j}=X_{i, j}-X_{i, j-1}$ denote the degradation increment for the $i$ th degradation process between two consecutive measurements. Let $\Delta t_{j}={ }_{j}-t_{j-1}$ be the time interval between two consecutive measurements. Owing to the independent increment property of the gamma process, we have

$$
\Delta X_{i, j} \sim G a\left(\Delta t_{j} ; \alpha_{i}, \beta_{i}\right)
$$

The likelihood function can then be obtained as

$$
L(\boldsymbol{\theta})=\prod_{j=1}^{J} f_{\Delta X_{1, j}, \Delta X_{2, j}, \ldots, \Delta X_{I, j}}\left(\Delta x_{1, j}, \Delta x_{2, j}, \ldots, \Delta x_{I, j} ; \Delta t_{j}\right)
$$

where $\boldsymbol{\theta}$ contains the marginal distribution parameters $\left(\alpha_{i}, \beta_{i}\right)$ for $i=1,2, \ldots, I$, and the copula parameter $\eta, \boldsymbol{\theta}=\left[\eta, \alpha_{1}, \beta_{1}, \alpha_{2}, \beta_{2, \ldots}, \alpha_{I}, \beta_{I}\right]$. Based on the joint distribution in Eq (6), we can rewrite the log-likelihood function as

$$
\begin{aligned}
l(\boldsymbol{\theta}) & =\sum_{j=1}^{J} c\left(F_{\Delta X_{1, j}}\left(\Delta x_{1, j} ; \Delta t_{j}\right), F_{\Delta X_{2, j}}\left(\Delta x_{2, j} ; \Delta t_{j}\right), \ldots, F_{\Delta X_{I, j}}\left(\Delta x_{I, j} ; \Delta t_{j}\right)\right) \\
& +\sum_{j=1}^{J} \sum_{i}^{I} f_{\Delta X_{i, j}}\left(\Delta x_{i, j} ; \Delta t_{j}\right)
\end{aligned}
$$

Maximum likelihood estimation (MLE) is employed to evaluate the parameters. However, 
since the number of parameters increases rapidly with dimensions, direct estimation using standard approaches such as maximum likelihood and moment estimation, may be impossible because of numerical difficulties (Renard and Lang, 2007). A common practice is to estimate the parameters of marginal distributions separately as a first step, and then estimate the copula parameters with the estimated marginal parameters, which is referred to as two-stage statistical inference for copula parameters (Wang and Pham, 2012). The marginal distribution parameters $\left(\alpha_{i}, \beta_{i}\right)$ can be estimated separately with the degradation data (Pan et al., 2011; Wang et al., 2015). Let $\kappa=\left[\alpha_{1}, \beta_{1}, \alpha_{2}, \beta_{2}, \ldots, \alpha_{I}, \beta_{I}\right]$ be the set of marginal parameters. After achieving the estimated $\kappa$, the copula parameters can be readily obtained as

$$
\hat{\eta}=\underset{\eta}{\arg \max } \sum_{j=1}^{J} c\left(F_{\Delta X_{1, j}}\left(\Delta x_{1, j} ; \Delta t_{j}\right), F_{\Delta X_{2, j}}\left(\Delta x_{2, j} ; \Delta t_{j}\right), \ldots, F_{\Delta X_{I, j}}\left(\Delta x_{I, j} ; \Delta t_{j}\right) ; \hat{\kappa}\right)
$$

\subsubsection{Special case: Bivariate degradation processes}

When the system is subject to bivariate degradation processes, the Frechet-Hoeffding bounds provide the upper and lower bounds of system reliability at time $t$ (Nelsen, 2007; Wang and Pham, 2012), i.e.,

$$
\begin{aligned}
& \max \left(F_{X_{1}}\left(\zeta_{1} ; t\right)+F_{X_{2}}\left(\zeta_{2} ; t\right)-1\right) \leq R(t) \\
& =C\left(F_{X_{1}}\left(\zeta_{1} ; t\right), F_{X_{2}}\left(\zeta_{2} ; t\right)\right) \leq \min \left(F_{X_{1}}\left(\zeta_{1} ; t\right), F_{X_{2}}\left(\zeta_{2} ; t\right)\right)
\end{aligned}
$$

In addition, Nelsen et al. (2001) reported a tighter reliability bound and provided the limit of reliability estimation in terms of two common dependence measures: Kendall's tau $\tau_{k}$ and Spearman's rho $\rho_{s}$. Concerning the Kendall's tau, we can have the upper bound as

$$
U_{\tau_{k}}(u, v)=\min \left(u, v, \frac{1}{2}\left(u+v-1+\sqrt{(u+v-1)^{2}+1+\tau_{k}}\right)\right)
$$


and the lower bound as

$$
L_{\tau_{k}}(u, v)=\max \left(0, u+v-1, \frac{1}{2}\left(u+v-\sqrt{(u-v)^{2}+1-\tau_{k}}\right)\right)
$$

where $u, v$ are the cdfs of marginal random variables, and

$$
\tau_{k}=4 \iint_{I^{2}} C(u, v) d C(u, v)-1
$$

The reliability bounds with respect to Spearman's rho are expressed as

$$
U_{\rho_{s}}(u, v)=\min \left(u, v, \frac{u+v-1}{2}+\varphi\left(u+v-1,1+\rho_{s}\right)\right)
$$

and

$$
L_{\rho_{s}}(u, v)=\max \left(0, u+v-1, \frac{u+v}{2}-\varphi\left(u-v, 1-\rho_{s}\right)\right)
$$

where

$$
\begin{gathered}
\rho_{s}=12 \iint_{I^{2}} C(u, v) d u d v-3 \\
\varphi(a, b)=\frac{1}{6}\left(\left(9 b+3 \sqrt{9 b^{2}-3 a^{6}}\right)^{1 / 3}+\left(9 b-3 \sqrt{9 b^{2}-3 a^{6}}\right)^{1 / 3}\right)
\end{gathered}
$$

Let $u=F_{X_{1}}\left(\zeta_{1} ; t\right)$ and $v=F_{X_{2}}\left(\zeta_{2} ; t\right)$. We can have the reliability bounds as

$$
L_{\tau_{k}}\left(F_{X_{1}}\left(\zeta_{1} ; t\right), F_{X_{2}}\left(\zeta_{2} ; t\right)\right) \leq R(t) \leq U_{\tau_{k}}\left(F_{X_{1}}\left(\zeta_{1} ; t\right), F_{X_{2}}\left(\zeta_{2} ; t\right)\right)
$$

with respect to Kendall's tau, and

$$
L_{\rho_{s}}\left(F_{X_{1}}\left(\zeta_{1} ; t\right), F_{X_{2}}\left(\zeta_{2} ; t\right)\right) \leq R(t) \leq U_{\rho_{s}}\left(F_{X_{1}}\left(\zeta_{1} ; t\right), F_{X_{2}}\left(\zeta_{2} ; t\right)\right)
$$


with respect to Spearman's rho.

\subsection{Reliability analysis considering environmental influence}

In this section, we consider the influence of the operating environment on the degradation processes and failure mechanism. It is assumed that the system is subject to multiple correlated degradation processes, in which the parameters of the degradation processes are usually estimated from test data by experiments (e.g., accelerated degradation test). When the system is put in use, the operating environment is different from that in experiments, which will exert influence on the degradation behaviours, especially the degradation rate. The environmental effect is hence used to distinguish the field use from experimental testing.

Environmental changes may accelerate or decelerate the deterioration process, and their influence is usually modeled as covariates (Deloux et al., 2009; Zhao et al., 2010). Given that the initial degradation level is $0, X_{i}(t)=0$ for $i \in\{1,2, \ldots, I\}$, the pdf of the degradation level at time $t$ is given as

$$
f_{X_{i}(t)}\left(x ; \alpha_{i}(t), \beta_{i}\right)=\frac{\beta_{i}^{\alpha_{i}(t)}}{\Gamma\left(\alpha_{i}(t)\right)} x^{\alpha_{i}(t)-1} e^{-\beta_{i} x}
$$

where $\alpha_{i}(t)$ is a time-dependent shape parameter. The environmental influence can be successfully characterised by choosing an appropriate $\alpha_{i}(t)$; usually, $\alpha_{i}(t)$ is given in the exponential or powerlaw form. Note that different choices of $\alpha_{i}(t)$ may comprise the stationary independent increment property, which turns out as a non-stationary gamma process (Van Noortwijk, 2009). If $\alpha_{i}(t)$ is linear, then the degradation process is reduced to the stationary gamma process as shown in Eq (1).

In addition, variations of the operating environment may affect the failure threshold. When operating in a changing environment, the system may fail before or after reaching the fixed failure threshold. In other words, the failure threshold can be random. Assume that the failure threshold 
$\zeta_{i}$ is an exponentially distributed random variable, i.e.,

$$
f_{\zeta_{i}}(s)=\frac{1}{\lambda_{i}} e^{-s / \lambda_{i}}
$$

The system fails when the degradation level hits the random threshold. For the $i$ th degradation process, the cumulative distribution of the failure time can be obtained as

$$
\begin{aligned}
F_{T_{i}}(t) & =P\left(X_{i}(t) \geq \zeta_{i}\right) \\
& =\int_{0}^{\infty} P\left(X_{i}(t) \geq s \mid \zeta_{i}=s\right) f_{\zeta_{i}}(s) d s \\
& =\frac{1}{\lambda_{i} \Gamma\left(\alpha_{i}(t)\right)} \int_{0}^{\infty} \gamma\left(\alpha_{i}(t), s \beta_{i}\right) e^{-s / \lambda_{i}} d s
\end{aligned}
$$

The assumption of an exponentially distributed random variable is made for mathematical simplicity. The method can be applied for other distributions as well. In Eq (14), the cumulative distribution of failure time can be obtained by replacing the pdf of the exponentially distributed random variable with the corresponding distribution. For a general distribution of the failure threshold, numerical methods can be used to compute the distribution of failure time. For the exponentially distributed failure threshold, by applying the Lindeberg-Feller central limit theorem, the failure distribution can be approximated as (Paroissin and Salami, 2014;, Park and Padgett, 2005)

$$
F_{T_{i}}(t) \approx 1-\left(1+1 /\left(\beta_{i} \lambda_{i}\right)\right)^{-\alpha_{i}(t)}
$$

The approximation of Eq (15) significantly reduces the computational burden of the failure distribution. Subsequently, system reliability can be obtained by using copulas, i.e.,

$$
R(t)=C\left(1-F_{T_{1}}(t), 1-F_{T_{2}}(t), \ldots, 1-F_{T_{I}}(t)\right)
$$

Note that Eq (16) constructs the copula function with respect to the failure time distribution of each 
degradation process instead of the distribution of degradation increments. This is due to the fact that system reliability is determined by both the random failure threshold and the property of the non-stationary gamma process, while the degradation data in isolation fails to provide enough information for the reliability evaluation. $\mathrm{Eq} \mathrm{(15)} \mathrm{and} \mathrm{(16)} \mathrm{indicate} \mathrm{that} \mathrm{we} \mathrm{can} \mathrm{evaluate} \mathrm{the} \mathrm{marginal}$ distribution of the failure time for each degradation process, and then obtain the overall system reliability using copula.

\section{Life cycle cost analysis}

This section presents the life cycle cost analysis with the renewal-cycle-based approach, which is the most widely used method for cost analysis in a finite horizon. Compared with Monte Carlo simulation, the renewal-cycle-based approach used in this paper can provide analytical expression for the expectation and variance of the cost. Moreover, the proposed approach is computationally efficient through the numerical example.

It is assumed that the system is perfectly maintained upon each failure. After maintenance, the system operates from the initial degradation level 0 , which constitutes a renewal cycle. The assumption of as good as new maintenance policy is used for two reasons. One is for mathematical simplicity. Based on the assumption of "as good as new" maintenance policy, the renewal cycle can be obtained, which is the interval between two failures/maintenance actions. It is quite difficult to have an analytical life-cycle cost for an imperfect maintenance. The other reason is that for some systems where replacement/overhaul is allowable, such as electronic products, or pipelines, it is reasonable to assume "as good as new" after each maintenance/replacement. Many literatures have assumed the as good as new?model in maintenance, and imperfect repair can definitely be a good point for future research. It should be noted that the assumption of "as good as new" intervention policy is applicable for specific systems where replacement or an overhaul is allowable. For systems like bridge, it is not so appropriate to have the "as good as new" assumption, since we 
will never replace a bridge. We have to be very careful when making this assumption.

The cost consists of two items: cost due to failures and the maintenance cost. Denote $C_{f}$ as the failure cost and $C_{m}$ the maintenance cost each time a failure occurs. The cost due to failure is associated with the degradation processes while the maintenance cost is largely determined by the decision makers. Here we assume that the maintenance cost is a fixed value. For the failure cost, since the failure cost is highly dependent on the failure mechanism and the severity of failure, which may vary each time, it is more appropriate to model the failure $\operatorname{cost} C_{f}$ as a random variable instead of a deterministic value. Let $C_{s}$ denote the cost associated with the maintenance and failureinduced cost, $C_{s}=C_{m}+C_{f}$, with the mean $\mu_{c}$ and standard deviation $\sigma_{c}$.

We consider a discounted life-cycle cost, since the time value of money reflects the discounting for a future expense. It is true that for a finite horizon, the life cycle cost can be evaluated without discounting. The discounting model is more flexible than the non-discounting model. When the discounting parameter is close to 0 , then the discounting model is able to achieve similar performance as the non-discounting model. Our model can be applied to the non-discounting case by setting the discount parameter to 0 . Let $K(t)$ denote the total cost in time interval $(0, t)$. Let $S_{i}$ be the time where the $i$ th renewal cycle ends, and the sequence $\left\{S_{0}, S_{1}, S_{2}, \ldots\right\}$ be the renewal process, where $S_{0}$ is the origin of the process. $K(t)$ can be expressed in terms of $S_{i}$ as

$$
K(t)=\sum_{i=1}^{\infty} C_{i} e^{-\rho S_{i}} \mathbf{1}_{\left\{S_{i} \leq t\right\}}
$$

where $C_{i}$ is the cost at the $i$ th renewal cycle, $\rho(\rho>0)$ is the discount rate and $\mathbf{1}_{\{\bullet\}}$ is the indicator function. In the following, we investigate the expectation and variance of the life cycle cost $K(t)$. Variance is an important factor in life cycle cost evaluation, because the expectation alone may suffer high uncertainty without indicating the variance. The concept of renewal decomposition is employed to calculate the expectation and variance of the life cycle cost (Pandey and van der Weide, 2017). The renewal decomposition refers to the basic property of renewal process that a 
probabilistic replica of the original process repeats after each renewal. In the present study, the degradation processes of the system renew at each failure/maintenance.

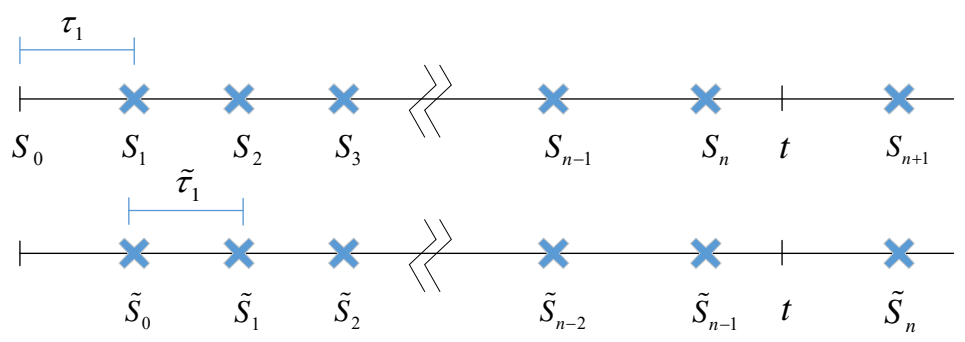

Figure 2: Description of renewal decomposition (original and shifted renewal process)

Figure 2 shows an original and a shifted renewal process in interval $(0, t)$ with the number of renewals $N(t)$. As shown in Figure 2, if the process is observed at the first event $S_{1}=\tau_{1}$, it is then shifted within the interval $\left(S_{1}, S_{1}+t\right)$. Let the occurrence time of the shifted renewal process be $\tilde{S}_{i}$. We have $\tilde{S}_{i}=S_{i+1}-\tau_{1}$. The number of renewal cycles in the shifted process is then given as

$$
\tilde{N}(t)=\sum_{i=1}^{\infty} \mathbf{1}_{\left\{\tilde{S}_{i} \leq t\right\}}
$$

It can be concluded that the distribution of $N(t)$ is identical to $\tilde{N}(t)$. In addition, the shifted process is independent of the observation time $\tau_{1}$. With the two properties, the discounted total $\operatorname{cost} K(t)$ of Eq (17) can be decomposed at the time of first renewal cycle, $S_{1}=\tau_{1}$, which leads to

$$
K(t)=C_{1} e^{-\rho \tau_{1}} \mathbf{1}_{\left\{\tau_{1} \leq t\right\}}+\sum_{i=1}^{\infty} C_{i+1} e^{-\rho S_{i+1}} \mathbf{1}_{\left\{\tilde{S}_{i} \leq t-\tau_{1}\right\}}
$$

Let

$$
\tilde{K}(t)=\sum_{i=1}^{\infty} C_{i} e^{-\rho \tilde{S}_{i}} \mathbf{1}_{\left\{\tilde{S}_{i} \leq t\right\}}
$$


It follows that

$$
K(t)=C_{1} e^{-\rho \tau_{1}} \mathbf{1}_{\left\{\tau_{1} \leq t\right\}}+e^{-\rho \tau_{1}} \tilde{K}\left(t-\tau_{1}\right)
$$

The expected discounted cost can be formulated as

$$
E[K(t)]=z(t)+\int_{0}^{t} e^{-\rho s} E\left[\tilde{K}\left(t-\tau_{1}\right)\right] d F_{T_{f}}(s)
$$

where $T_{f}$ is the time to failure of the system, $T_{f}=\min \left\{T_{1}, T_{2}, \ldots, T_{I}\right\}, F_{T_{f}}(t)=1-R(t)$, and

$$
z(t)=E\left[C_{1} e^{-\rho \tau_{1}} \mathbf{1}_{\left\{\tau_{1} \leq t\right\}}\right]
$$

By solving the above integral equation, we can have the expected discounted cost as

$$
E[K(t)]=z(t)+\int_{0}^{t} z(t-s) e^{-\rho s} d \Lambda(s)
$$

where $\Lambda(t)$ is the renewal function,

$$
\Lambda(t)=E[N(t)]=F_{T_{f}}(t)+\int_{0}^{t} \Lambda(t-u) d F_{T_{f}}(u)
$$

The renewal function can be numerically solved by the RS-method following definition of the Riemann-Stieltjes integration (Xie, 1989).

Since the expectation of life-cycle cost can be similar for a degradation process with high volatility and that with low volatility, it fails to characterize the stochasticity of the degradation processes. Variance, to some extent, can characterize the volatility of the cost, which contributes to the decision makings, such as allocating budgets and developing maintenance policies. For example, in the mean-variance?based utility framework, the decision makers are able to evaluate the cost together with the associated risk, which provides more alternatives in terms of the risk 
attitudes of the decision makers.

Based on Eq (19), the second moment of the discounted cost can be obtained as

$$
\begin{aligned}
E\left[K^{2}(t)\right] & =E\left[\left(C_{1} e^{-\rho \tau_{1}} \mathbf{1}_{\left\{\tau_{1} \leq t\right\}}+\sum_{i=1}^{\infty} C_{i+1} e^{-\rho S_{i+1}} \mathbf{1}_{\left\{\tilde{S}_{i} \leq t-T_{1}\right\}}\right)^{2}\right] \\
& =\chi(t)+\int_{0}^{t} e^{-2 \rho s} E\left[K^{2}(t-s)\right] d F_{T_{f}}(s)
\end{aligned}
$$

where

$$
\chi(t)=E\left[\left(C_{1}^{2}+2 C_{1} \tilde{K}\left(t-\tau_{1}\right)\right) e^{-2 \rho \tau_{1}} \mathbf{1}_{\left\{\tau_{1} \leq t\right\}}\right]
$$

Similarly, by using renewal decomposition, the solution of Eq (22) can be obtained as

$$
E\left[K^{2}(t)\right]=\chi(t)+\int_{0}^{t} e^{-2 \rho s} \chi(t-s) d \Lambda(s)
$$

Combining Eq (21) and Eq (23), we can have the variance of the discounted cost as

$$
\begin{aligned}
\operatorname{Var}(K(t)) & =E\left[K^{2}(t)\right]-E[K(t)]^{2} \\
& =\chi(t)+\int_{0}^{t} e^{-2 \rho s} \chi(t-s) d \Lambda(s) \\
& -\left(z(t)+\int_{0}^{t} z(t-s) e^{-\rho s} d \Lambda(s)\right)^{2}
\end{aligned}
$$

If the cost item $C_{s}$ is independent of the system failure time $T_{f}$, the expectation of the discounted cost is reduced to

$$
E[K(t)]=\mu_{c} \int_{0}^{t} e^{-\rho s} d \Lambda(s)
$$

and the second moment is given as

$$
E\left[K^{2}(t)\right]=\left(\mu_{c}^{2}+\sigma_{c}^{2}\right) \int_{0}^{t} e^{-2 \rho s} d \Lambda(s)+2 \mu_{c} \int_{0}^{t} e^{-2 \rho s} E[K(t-s)] d \Lambda(s)
$$


Then the variance is expressed as

$$
\begin{aligned}
\operatorname{Var}(K(t)) & =\left(\mu_{c}^{2}+\sigma_{c}^{2}\right) \int_{0}^{t} e^{-2 \rho s} d \Lambda(s)+2 \mu_{c} \int_{0}^{t} e^{-2 \rho s} E[K(t-s)] d \Lambda(s) \\
& -\mu_{c}^{2}\left(\int_{0}^{t} e^{-\rho s} d \Lambda(s)\right)^{2}
\end{aligned}
$$

\section{Numerical example}

In this section, we present an illustrative example to show the system reliability modelled by a copula function and the life cycle cost thereafter. The system is subject to two degradation processes, modelled by gamma processes. The Clayton copula is used to describe the dependence of the degradations. The bivariate Clayton copula is given as

$$
C(u, v ; \eta)=\max \left(\left(u^{-\eta}+v^{-\eta}-1\right)^{-1 / \eta}, 0\right)
$$

with the density function

$$
c(u, v ; \eta)=\frac{\partial C(u, v ; \eta)}{\partial \eta}=\frac{(1+\eta)(u v)^{-1-\eta}}{\left(u^{-\eta}+v^{-\eta}-1\right)^{\frac{1}{\eta}+2}}
$$

To illustrate the parameter estimation procedure for the marginal distribution parameters and copula parameters, a set of simulated data are generated as a first step. Thanks to Sklar's theorem, we only need to generate a sample pair $(u, v)$ from uniform random variables $(0,1)$ whose joint distribution is $C(u, v)$, and then transform the $(u, v)$ to degradation observations $\left(\Delta x_{1}, \Delta x_{2}\right)$ by the inverse distribution function. The conditional distribution method serves for the data generation purpose (Pan et al., 2011). Given $U=u$, the conditional distribution of $V$ is expressed as

$$
c_{c}(v)=P(V \leq v \mid U=u)=\frac{\partial C(u, v ; \eta)}{\partial u}
$$

The procedure to generate the degradation observations $\left(\Delta x_{1}, \Delta x_{2}\right)$ can be summarised as fol- 
lows:

1. Generate two uniform $(0,1)$ variates $u_{1}$ and $v_{0}$ independently.

2. Let $u_{2}=c_{c}^{-1}\left(v_{0}\right)$, where $c_{c}^{-1}$ is the quasi-inverse of $c_{c}$.

3. Let $\Delta x_{i}=F_{\Delta X_{i}}^{-1}\left(u_{i}\right), i=1,2$, where $F_{\Delta X_{i}}^{-1}$ the quasi-inverse of $F_{\Delta X_{i}}$. Then $\left(\Delta x_{1}, \Delta x_{2}\right)$ is the desired degradation observations.

The true values of the degradation parameters for data simulation are set as $\alpha_{1}=\alpha_{2}=1$, $\beta_{1}=\beta_{2}=2$, and $\eta=1$. In total, 1,000 data sets are generated based on the generation procedure, which are further used for parameter estimation. The time interval of the degradation observations is set as 1 .

\subsection{Parameter \& system reliability estimation}

Maximum likelihood estimation is employed to estimate the marginal and copula parameters. The detailed estimation procedure of the parameters is provided in Section 2.2.1. The parameters are estimated with the simulated degradation data, of which the underlying parameters are summarised in Table 1. Note the in the following analysis of this section, the results are obtained in terms of the estimates. For illustration purpose, we present the 3D and contour plot of the Clayton copula in Figure 3.

Table 1: Estimation of the parameters

\begin{tabular}{llll}
\hline Parameters & True value & Estimated value & Relative error(\%) \\
$\alpha_{1}$ & 1 & 1.0307 & 3.07 \\
$\beta_{1}$ & 2 & 1.8833 & 5.835 \\
$\alpha_{2}$ & 1 & 1.0254 & 2.54 \\
$\beta_{2}$ & 2 & 1.9419 & 2.905 \\
$\eta$ & 1 & 0.8827 & 11.63 \\
\hline
\end{tabular}



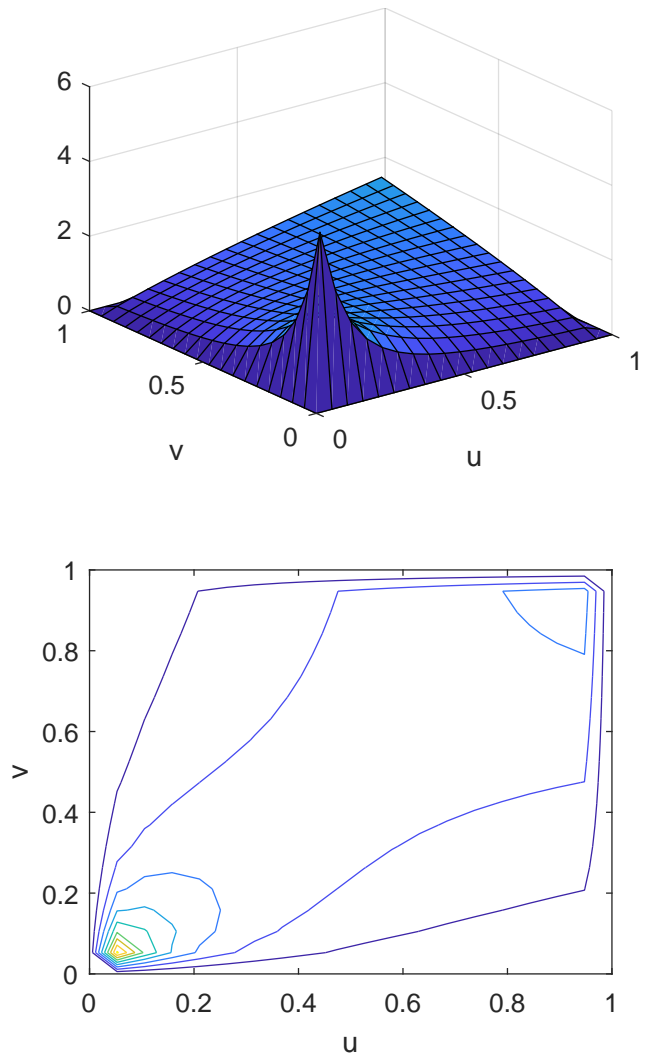

Figure 3: Pdf plot of Clayton copula with $\eta=0.8827$ 
The system fails when either of the degradation levels hits the threshold $\zeta_{i}=30$, for $i=1,2$. System reliability can be obtained based on Eq (7). Figure 4 presents the cdf plot of system failure time $T_{f}$ and the marginal distributions $T_{1}, T_{2}$. It can be observed that system failure occurs within the time interval $(30,80)$, while the mean time to failure is 50.35 . To better illustrate the failure time, we sample from the reliability distribution and plot histogram of the failure time in Figure 5.

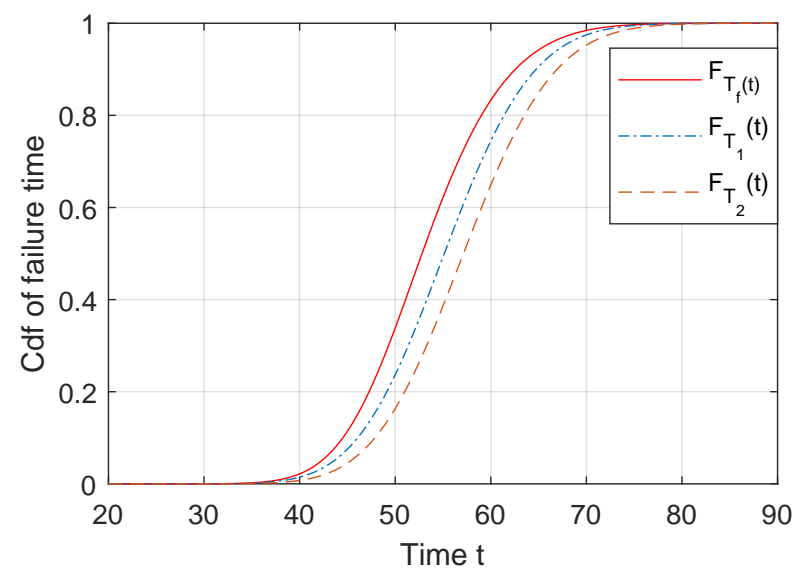

Figure 4: Cdf plot of system $T_{f}, T_{1}$ and $T_{2}$

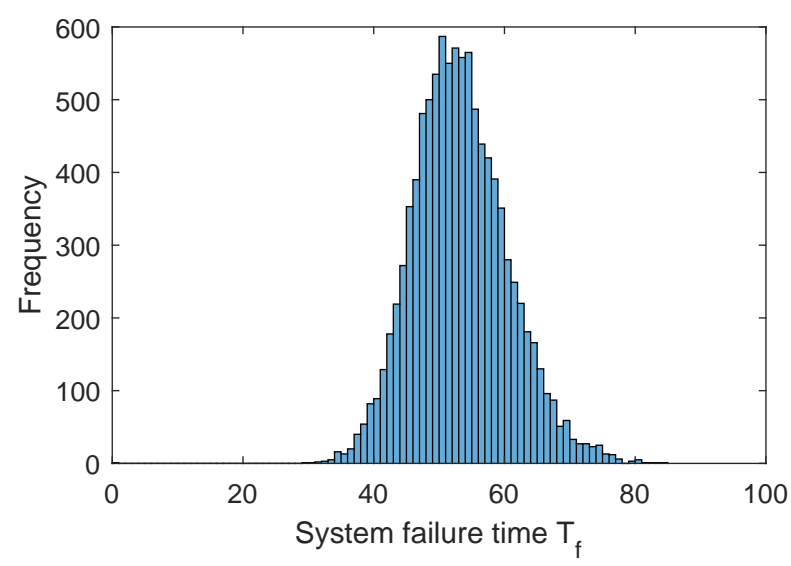

Figure 5: Histogram of sampled failure time

In addition, as previously discussed, the range of bivariate copula functions can be evaluated by the Frechet-Hoeffding bounds, which apply to system reliability estimation regardless of the 
form of copula functions. The Frechet-Hoeffding bounds are robust for uncertainty modelling because of their applicability to various copulas. However, the provided upper and lower bounds are not as tight as other bounds. A tighter bound can be achieved with respect to Kendall's tau or Spearman's rho, in presence of a measure of association and marginal distributions. Figure 6 shows the variation of system reliability and the associated upper and lower bounds in terms of Kendall's tau.

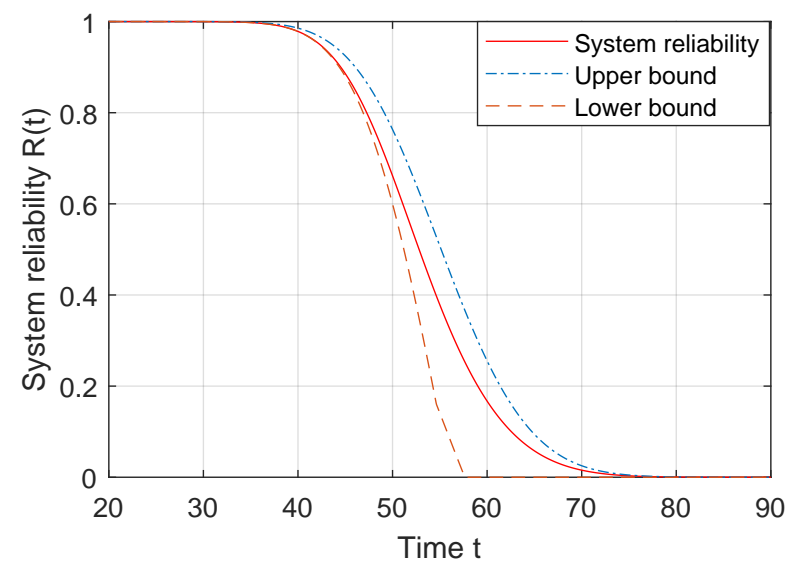

Figure 6: Bounds of system reliability in terms of Kendall's tau

\subsection{System reliability with environmental influence}

In this section we analyse the system reliability in presence of environmental influence. As previously stated, the environmental influence lies in accelerating/decelerating the degradation process and randomising the failure threshold. We use a power-law parameter to characterise the degradation rate, $\alpha_{i}(t)=\alpha_{i} t^{\omega}$. Note that $\omega>1$ implies degradation acceleration while $\omega<1$ indicates deceleration. We set the parameter as $\omega=1.2$. To facilitate comparison with the previous results, we let $\alpha_{1}=1.0307$ and $\alpha_{2}=1.0254$. Other parameters are identical to the estimated values in Section 4.1. With the environmental influence, the failure threshold is stochastic rather than deterministic. We assume that the failure threshold follows an exponential distribution, with the 
parameter $\lambda_{1}=\lambda_{2}=30$.

Figure 7 shows the system reliability with environmental influence, along with the marginal bounds of the Kendall's tau and marginal distributions of the two degradation processes. Figure 7 presents the integrated impact of random failure thresholds and degradation acceleration.
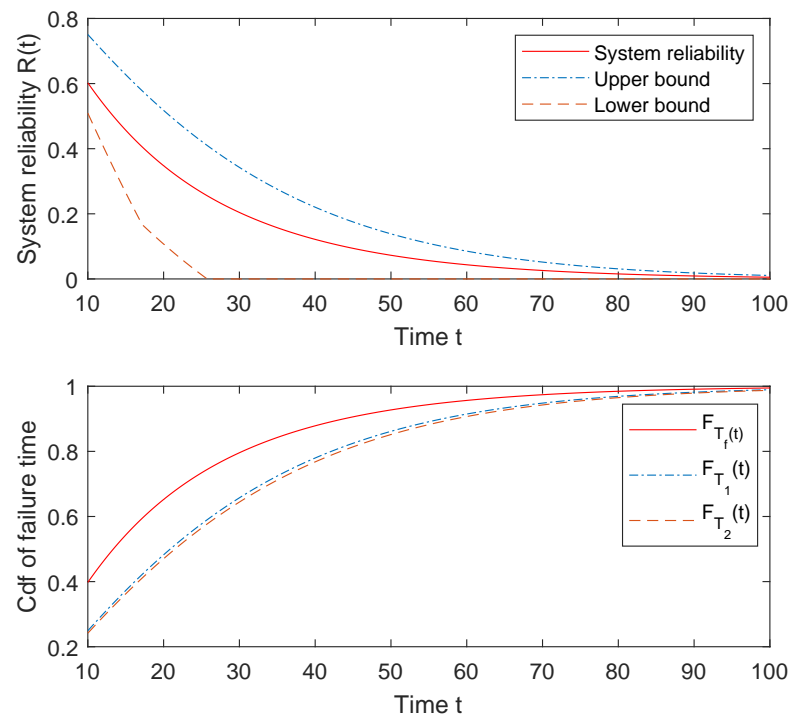

Figure 7: Plot of system reliability, Kendall's tau and marginal distributions with environmental influence

To investigate the influence of random failure thresholds and degradation acceleration separately, we plot the system reliability with deterministic thresholds in Figure 8 for comparison. Observe that that the reliability curves from Figure $8(a, b)$ and Figure 6 are quite different, which implies the significant influence of random failure thresholds. Further, the environmental influence on degradation acceleration can be observed by comparing the reliability variations of Figure 6 and Figure $8(c, d)$. It can be observed that in the presence of the accelerated degradation, the system reliability decreases faster than the homogeneous gamma deterioration process. In addition, the reliability curve is shifted to the left. The observations are intuitive, due to the fact that the existence of environmental influence accelerates the degradation processes when the power-law parameter $\omega>1$. 

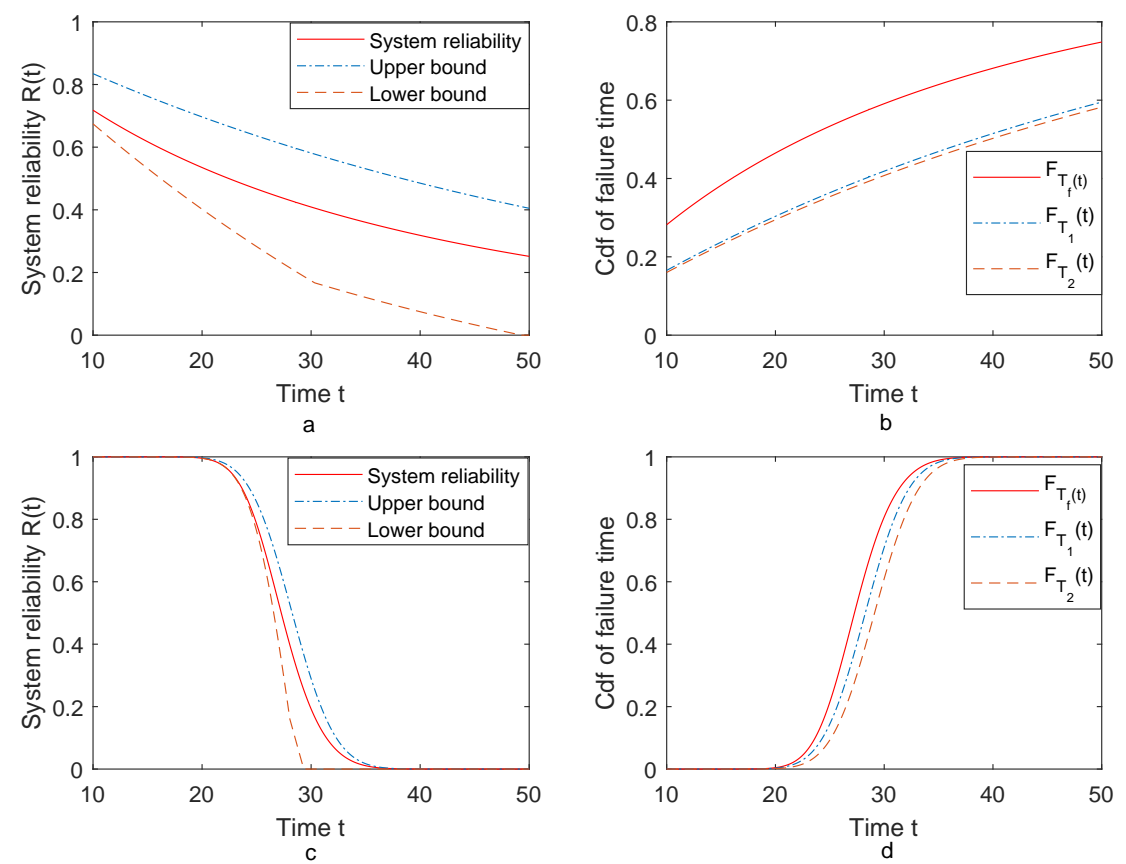

Figure 8: Plot of $(\mathrm{a}, \mathrm{b})$ system reliability, Kendall's tau and marginal distributions with random failure thresholds and homogeneous degradation, (c,d) with deterministic failure thresholds and accelerated degradation 
Under the current setting, the mean time to failure is 18.6, which is quite different from that without environmental influence. We are interested in the parameters $\lambda_{i}$ and $\omega$, since $\lambda_{i}$ implies the influence of a random failure threshold and $\omega$ stands for the impact of degradation acceleration/deceleration. In Figure 9, we plot the variation of mean time to failure and system lifetime with respect to $\lambda_{i}$ and $\omega$. It can be observed that both the mean time to failure and system reliability increases with $\lambda_{i}$ and decreases with $\omega$. This is due to the fact that a large $\lambda_{i}$ increases the failure threshold (randomly) while a large $\omega$ leads to a rapid deterioration process. Significantly, Figure $9(d)$ actually presents the system reliability under decelerated $(\omega=0.8)$, homogeneous $(\omega=1)$ and accelerated $(\omega=1.2)$ degradation processes.
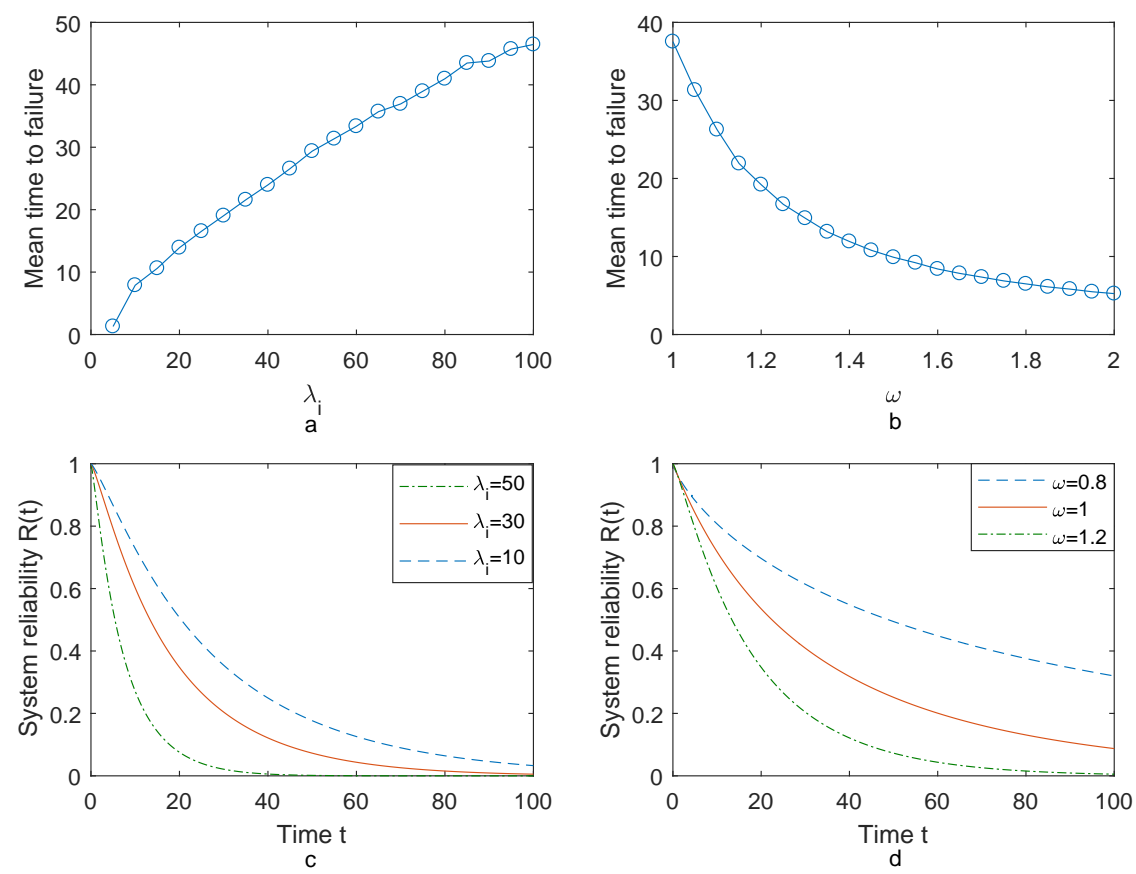

Figure 9: Influence of $\lambda_{i}$ and $\omega$ on $(a, b)$ mean time to failure and (c,d) system reliability

\subsection{Life cycle cost analysis}

The system is perfectly maintained (perfect repair or replacement) at failure, which constitutes a renewal cycle. We consider a discounted cost since the value of money decreases with time. 
We consider the case that the cost at each failure is independent from the system failure time. As discussed in Section 3, we do not need to specify the distribution of the cost for life cycle analysis; what required is the mean and standard deviation. In the present example, the mean and standard deviation of the cost $C_{s}$ are given as $\mu_{c}=10$ and $\sigma_{c}=5$. Figure 10 presents the expectation, variance and second moment of the life cycle cost, with the discounted rate $\rho=0.005$. In addition, we compare the analytical result with that by Monte Carlo simulation in Figure 10. It should be noted that numerical methods are used to compute the life cycle cost in Section 3 . The numerical method here refers to discretising the continuous variables into discrete ones. Monte Carlo simulation here indicates to repeatedly simulate the failure time and total cost within the operating horizon for multiple times, and then obtain the expected cost and variance from the set of simulated cost. The numerical method here requires the analytical expression of the life cycle cost, while Monte Carlo simulation does not. As can be observed, both the expected cost $E[K(t)]$ and second moment $E\left[K^{2}(t)\right]$ increase with time. It is worth noting that $\operatorname{Var}(K(t))$ is close to or even higher than the expected cost $E[K(t)]$ during the time interval $(100,500)$, which indicates that cost estimation purely based on expected cost will lead to a meaningless result owing to the high volatility of life cycle cost. Decision-makers are suggested to incorporate the uncertainty of life cycle cost when making investment decisions.

The reason for such a high variance is that the life cycle cost is subject to uncertainties: One is the intrinsic randomness due to the stochastic degradation process, and the other is the epistemic uncertainty in evaluating the maintenance and failure cost.

Figure 11 shows how the life cycle cost varies with the operating time considering the environmental influence. Notably, the expectation and variance of the life time cost are much larger than those without environmental influence. This is due to the fact that system lifetime has been significantly reduced in the way that the operating environment has accelerated the degradation process and randomised the failure thresholds. Therefore, we conclude that environmental influence contributes significantly to the lifetime cost. We recommend that maintenance managers take 

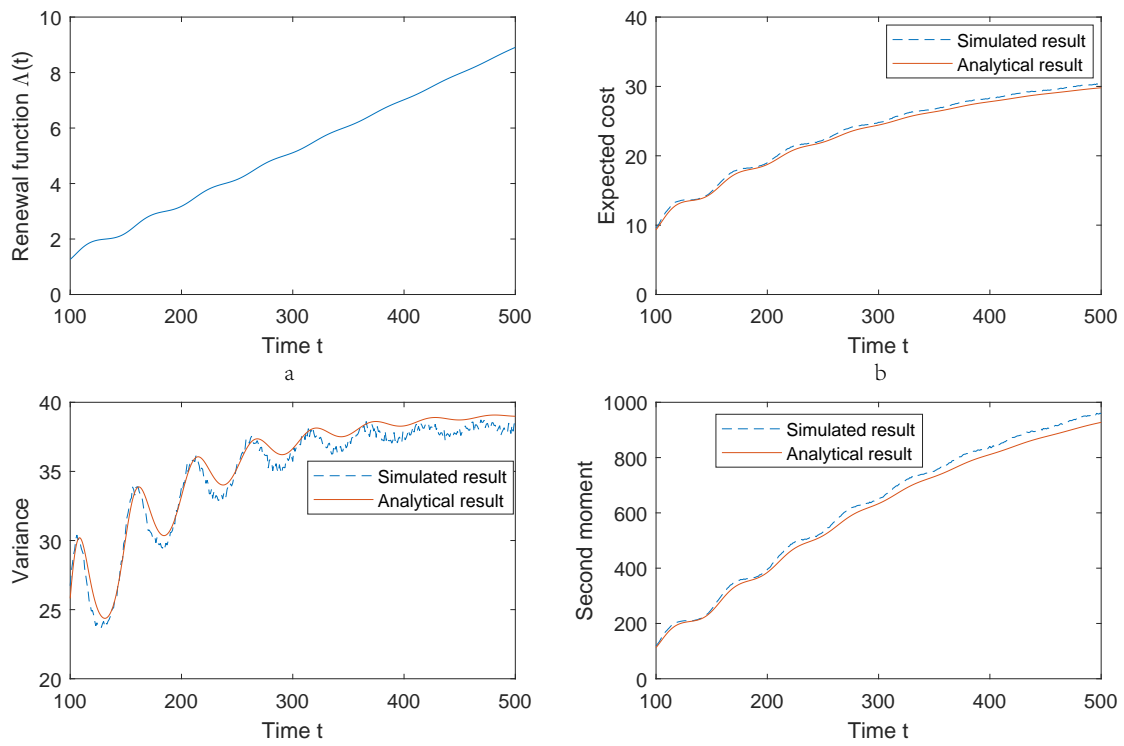

Figure 10: Life cycle cost without environmental influence: (a) renewal function, (b) expected cost, (c) variance and (d) second moment

effective measures to diminish its effect.

The proposed model can be applied to real examples, such as electronic products and pipelines. As shown in the numerical example, data is where we start from. Based on the simulated data, we estimate the associated parameters and the life cycle cost. For a real example, what we need is the data for the degradation processes. As long as the degradation data are provided, we can evaluate system reliability and life cycle cost with the proposed model.

\section{Conclusions}

In this study, we established a life cycle cost model for systems with multiple dependent degradation processes and environmental influence. We evaluated the expectation and variance of the life cycle cost for a finite horizon. Copula functions were employed to characterise the dependence of degradation processes, which serves for system reliability and the subsequent life cycle cost analysis. The operating environment affects system reliability in such a way that it acceler- 

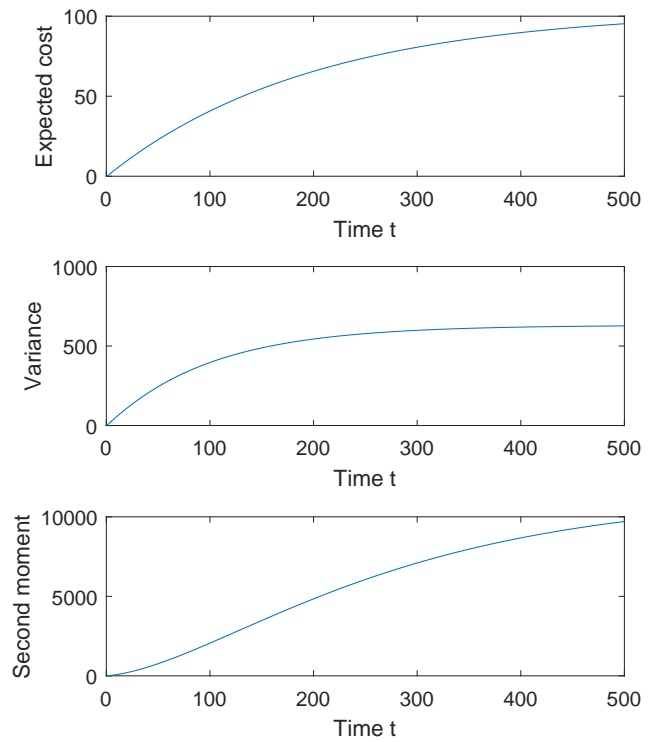

Figure 11: Life cycle cost with environmental influence: expected cost, variance and second moment

ates/decelerates the degradation process and leads to random failure thresholds. We show through a numerical example that the operating environment can have significant impacts on system reliability and life cycle cost. We suggest that engineers take effective measures to diminish the environmental influence and prolong system lifetime.

Future research can be conducted in the following two directions. First, in the current study we evaluated the life cycle cost focusing on the renewal of failures, without incorporating system intervention. Future research can consider system interventions, such as preventive maintenance or adding more redundancies. Specifically, condition-based maintenance and predictive maintenance strategies can be implemented to prevent system failure. Second, the environmental influence on the degradation process and system reliability can be investigated in detail. We assumed exponentially distributed failure thresholds under environmental influence. For different systems, the influence may vary with the operating environment and failure mechanisms. An accurate description of the environmental influence can be achieved using field data and expert knowledge. 


\section{References}

Caballé, N. and Castro, I. (2017), 'Analysis of the reliability and the maintenance cost for finite life cycle systems subject to degradation and shocks', Applied Mathematical Modelling 52, 731746.

Deloux, E., Castanier, B. and Bérenguer, C. (2009), 'Predictive maintenance policy for a gradually deteriorating system subject to stress', Reliability Engineering \& System Safety 94(2), 418-431.

Do, P., Assaf, R., Scarf, P. and Iung, B. (2019), 'Modelling and application of condition-based maintenance for a two-component system with stochastic and economic dependencies', Reliability Engineering \& System Safety 182, 86-97.

Frangopol, D. M. (2011), 'Life-cycle performance, management, and optimisation of structural systems under uncertainty: accomplishments and challenges 1', Structure and Infrastructure Engineering 7(6), 389-413.

Gao, H., Cui, L. and Qiu, Q. (2019), 'Reliability modeling for degradation-shock dependence systems with multiple species of shocks', Reliability Engineering \& System Safety 185, 133143.

Gebraeel, N. and Pan, J. (2008), 'Prognostic degradation models for computing and updating residual life distributions in a time-varying environment', IEEE Transactions on Reliability 57(4), 539-550.

Hong, H., Zhou, W., Zhang, S. and Ye, W. (2014), 'Optimal condition-based maintenance decisions for systems with dependent stochastic degradation of components', Reliability Engineering \& System Safety 121, 276-288.

Jiang, L., Feng, Q. and Coit, D. W. (2012), 'Reliability and maintenance modeling for dependent 
competing failure processes with shifting failure thresholds', IEEE Transactions on Reliability 61(4), 932-948.

Liu, B., Liang, Z., Parlikad, A. K., Xie, M. and Kuo, W. (2017), 'Condition-based maintenance for systems with aging and cumulative damage based on proportional hazards model', Reliability Engineering \& System Safety 168, 200-209.

Liu, B., Wu, S., Xie, M. and Kuo, W. (2017), 'A condition-based maintenance policy for degrading systems with age-and state-dependent operating cost', European Journal of Operational Research 263(3), 879-887.

Liu, B., Yeh, R.-H., Xie, M. and Kuo, W. (2017), 'Maintenance scheduling for multicomponent systems with hidden failures', IEEE Transactions on Reliability 66(4), 1280-1292.

Liu, X., Al-Khalifa, K. N., Elsayed, E. A., Coit, D. W. and Hamouda, A. S. (2014), 'Criticality measures for components with multi-dimensional degradation', IIE Transactions 46(10), 987998.

Liu, X., Li, J., Al-Khalifa, K. N., Hamouda, A. S., Coit, D. W. and Elsayed, E. A. (2013), 'Condition-based maintenance for continuously monitored degrading systems with multiple failure modes', IIE Transactions 45(4), 422-435.

Lu, C. J. and Meeker, W. O. (1993), 'Using degradation measures to estimate a time-to-failure distribution', Technometrics 35(2), 161-174.

Nelsen, R. B. (2007), An introduction to copulas, Springer Science \& Business Media.

Nelsen, R. B., Quesada-Molina, J. J., Rodriíguez-Lallena, J. A. and Ubeda-Flores, M. (2001), 'Bounds on bivariate distribution functions with given margins and measures of association', Communications in Statistics-Theory and Methods 30(6), 1055-1062. 
Pan, Z., Balakrishnan, N. and Sun, Q. (2011), 'Bivariate constant-stress accelerated degradation model and inference', Communications in Statistics-Simulation and Computation 40(2), 247257.

Pan, Z., Balakrishnan, N., Sun, Q. and Zhou, J. (2013), 'Bivariate degradation analysis of products based on wiener processes and copulas', Journal of Statistical Computation and Simulation 83(7), 1316-1329.

Pandey, M., Cheng, T. and van der Weide, J. (2011), 'Finite-time maintenance cost analysis of engineering systems affected by stochastic degradation', Proceedings of the Institution of Mechanical Engineers, Part O: Journal of Risk and Reliability 225(2), 241-250.

Pandey, M. D. and van der Weide, J. (2017), 'Stochastic renewal process models for estimation of damage cost over the life-cycle of a structure', Structural Safety 67, 27-38.

Park, C. and Padgett, W. (2005), 'Accelerated degradation models for failure based on geometric brownian motion and gamma processes', Lifetime Data Analysis 11(4), 511-527.

Paroissin, C. and Salami, A. (2014), 'Failure time of non homogeneous gamma process', Сотmunications in Statistics-Theory and Methods 43(15), 3148-3161.

Peng, W., Li, Y.-F., Yang, Y.-J., Zhu, S.-P. and Huang, H.-Z. (2016), 'Bivariate analysis of incomplete degradation observations based on inverse gaussian processes and copulas', IEEE Transactions on Reliability 65(2), 624-639.

Qiu, Q., Cui, L. and Kong, D. (2018), 'Availability and maintenance modeling for a two-component system with dependent failures over a finite time horizon', Proceedings of the Institution of Mechanical Engineers, Part O: Journal of Risk and Reliability p. 1748006X18768713.

Renard, B. and Lang, M. (2007), 'Use of a gaussian copula for multivariate extreme value analysis: some case studies in hydrology', Advances in Water Resources 30(4), 897-912. 
Riascos-Ochoa, J., Sánchez-Silva, M. and Klutke, G.-A. (2016), ‘Modeling and reliability analysis of systems subject to multiple sources of degradation based on lévy processes', Probabilistic Engineering Mechanics 45, 164-176.

Rosenblueth, E. and Mendoza, E. (1971), 'Reliability optimization in isostatic structures', Journal of the Engineering Mechanics Division 97(6), 1625-1642.

Sanchez-Silva, M., Klutke, G.-A. and Rosowsky, D. V. (2011), 'Life-cycle performance of structures subject to multiple deterioration mechanisms', Structural Safety 33(3), 206-217.

Sánchez-Silva, M., Klutke, G.-A. et al. (2016), Reliability and life-cycle analysis of deteriorating systems, Vol. 182, Springer.

Song, S., Coit, D. W., Feng, Q. and Peng, H. (2014), 'Reliability analysis for multi-component systems subject to multiple dependent competing failure processes', IEEE Transactions on Reliability 63(1), 331-345.

Van Noortwijk, J. (2009), 'A survey of the application of gamma processes in maintenance', Reliability Engineering \& System Safety 94(1), 2-21.

Wang, X., Balakrishnan, N., Guo, B. and Jiang, P. (2015), 'Residual life estimation based on bivariate non-stationary gamma degradation process', Journal of Statistical Computation and Simulation 85(2), 405-421.

Wang, Y. and Pham, H. (2012), 'Modeling the dependent competing risks with multiple degradation processes and random shock using time-varying copulas', IEEE Transactions on Reliability 61(1), 13-22.

Wang, Z., Huang, H.-Z., Li, Y. and Xiao, N.-C. (2011), 'An approach to reliability assessment under degradation and shock process', IEEE Transactions on Reliability 60(4), 852-863. 
Wu, S. (2014), 'Construction of asymmetric copulas and its application in two-dimensional reliability modelling', European Journal of Operational Research 238(2), 476-485.

Xie, M. (1989), 'On the solution of renewal-type integral equations', Communications in StatisticsSimulation and Computation 18(1), 281-293.

Yang, L., Zhao, Y., Peng, R. and Ma, X. (2018), 'Hybrid preventive maintenance of competing failures under random environment', Reliability Engineering \& System Safety 174, 130-140.

Ye, Z. S., Tang, L. C. and Xu, H. Y. (2011), 'A distribution-based systems reliability model under extreme shocks and natural degradation', IEEE Transactions on Reliability 60(1), 246-256.

Ye, Z.-S. and Xie, M. (2015), 'Stochastic modelling and analysis of degradation for highly reliable products', Applied Stochastic Models in Business and Industry 31(1), 16-32.

Zhai, Q. and Ye, Z.-S. (2017), 'Rul prediction of deteriorating products using an adaptive wiener process model', IEEE Transactions on Industrial Informatics 13(6), 2911-2921.

Zhao, X., Fouladirad, M., Bérenguer, C. and Bordes, L. (2010), 'Condition-based inspection/replacement policies for non-monotone deteriorating systems with environmental covariates', Reliability Engineering \& System Safety 95(8), 921-934.

Zhao, X., Xu, J. and Liu, B. (2018), 'Accelerated degradation tests planning with competing failure modes', IEEE Transactions on Reliability 67(1), 142-155. 